\title{
Scheufele, Bertram; Hummel, Gerrit; Rang, Henrik K.; Jost, Arietta; Satinsky, Pia; Wappler, Carolin: Der kommunikative Kosmos von Gefangenen. Eine sozialkonstruktivistische Studie zum Strafvollzug in Baden-Württemberg
}

Baden-Baden: Nomos 2019. 185 Seiten. Preis: $€ 39$

\author{
Maren Hartmann \\ Online publiziert: 11. März 2020 \\ (C) Der/die Autor(en) 2020
}

Dieses wichtige Buch präsentiert Forschung, die unter sehr schwierigen Bedingungen entstanden ist und gewährt damit einen ,Einblick in einen von der Bevölkerung meist kaum beachteten Teil der Gesellschaft" (S. 68). So entsteht ein kommunikationswissenschaftlicher Zugriff auf den kommunikativen Kosmos Strafgefangener. Zugleich liest sich das Buch wie eine Anleitung für diese Art von Forschung, denn es bietet eine beispielhafte Begründung des methodischen Vorgehens, der sozialkonstruktivistischen Herangehensweise und der typologisierenden Auswertung in einer Sorgfalt und Systematik, die seinesgleichen sucht. Geleitet wurde die Studie von Bertram Scheufele, durchgeführt von mehreren wissenschaftlichen und studentischen Mitarbeiter*innen. Dazu wurden 17 Leitfadengespräche mit Gefangenen der JVA Heimsheim in Baden-Württemberg geführt.

Ungewöhnlich an diesem Werk ist der Einstieg über ein Kapitel zu Reichweite und Perspektiven. Ausführlich hergeleitet wird dabei, warum die ( $\mathrm{Re}-)$ Konstruktion von Konstruktionen der gewählte Zugang ist und dass die Kommunikationswissenschaft als Bezugswissenschaft der Kriminologie dienen sollte. Bereits hier wird deutlich, dass es den Autor*innen sehr wichtig war, das Vorgehen des Projekts ausführlich zu begründen - und letztendlich auch zu verteidigen. Dazu gehört auch, dass das zweite Kapitel den Forschungsstand zur Mediennutzung im Strafvollzug darstellt und das dritte Kapitel dies dann wiederum auf den kommunikativen Kosmos ausweitet (dazu gehören jeweils die Rahmenbedingungen, eine Begriffsklärung - Medien einerseits, Kommunikation und Interaktion andererseits - und der Forschungsstand).

Medien spielen im Strafvollzug eine durchaus große Rolle, sei es zur Identitätsbildung (bzw. insbesondere deren Erhalt), zum Zeitvertreib oder aber zur Wei-

Prof. Dr. M. Hartmann ( $\square)$

Institut für Theorie und Praxis der Kommunikation, Universität der Künste Berlin,

Mierendorffstr. 30, 10589 Berlin, Deutschland

E-Mail: hartmann@udk-berlin.de 
terbildung. Der konzis zusammengefasste Forschungsstand weist auf offene Fragen hin, zum Beispiel zur gruppenspezifischen Abgrenzung (welche Motive der Mediennutzung sind spezifisch für Strafvollzugsgefangene?). Viele bisherige Erkenntnisse bezögen sich auch nur auf einzelne Medien (insbesondere Fernsehen). Die Autor*innen kritisieren zudem, dass es an multivariaten Perspektiven fehlt und es ein Generalisierungsproblem gibt. Insbesondere letzteres versuchen sie in ihrer Studie zu vermeiden.

Der kommunikative Kosmos Strafgefangener, so wird deutlich, umfasst die interpersonale Face-to-face Kommunikation, die vermittelte interpersonale Kommunikation und die massenmedial vermittelte Kommunikation - und all dies sowohl in der unmittelbaren Sozialwelt in der Haft, in der Sozialwelt vor und nach der Haft als auch in der mittelbaren Umwelt. Die Rahmenbedingungen der Kommunikation und Interaktion wiederum reichen von Arbeit über therapeutische Hilfe bis hin zur Regelung der Außenkontakte (primär mit Familie und Verteidiger) und umfassen auch die - durchaus restriktiven - rechtlichen Rahmenbedingungen. Die Autor*innen schaffen es hier, sich einerseits auf bestehende kommunikationswissenschaftliche Ansätze zu beziehen als auch die Spezifik der gewählten Umgebung bzw. Personengruppe aufzuzeigen.

Letztere tauchen abstrahiert wiederum im Kernkapitel des Buches auf. Dabei werden fünf Typologien (mit jeweils mehreren Typen) entwickelt. Den Forschungsfragen folgend beziehen sich die Typen auf die Konstruktion sinnstiftender Momente einerseits und die kommunikativen Verhältnisse zu vier unterschiedlichen Bezugsgruppen andererseits.

Beispielhaft genannt sei hier in der Typologie des kommunikativen Verhältnisses zu den Vollzugsbeamten der Typus des Gefangenen „Der Kritische“: Er zeichnet sich durch eingeschränkte Kommunikation aus (er führt nur oberflächliche Gespräche mit den Beamten), die vor allem der negativen Haltung gegenüber diesen geschuldet ist (die anderen drei Typen in dieser Typologie sind „,Der Distanzierte,“ „Der Differenzierende“ und „Der Strategische“). Bereits an diesem Typ zeigt sich die Verwobenheit von Kommunikation und Interaktion in der Spezifik des Strafvollzugs. Deutlich wird auch: Dieser Typ hätte mit einem Fokus „nur“ auf Medienkommunikation nicht entwickelt werden können. Diese Form der Kommunikation aber ist wiederum für andere Bezugsgruppen sehr zentral. In der Gesamtanalyse entsteht so eine typologische Kartographierung und damit eine durchaus facettenreiche Analyse des kommunikativen Kosmos' Strafgefangener bei bewusstem Verzicht auf konkrete Handlungsanweisungen.

Bei all der Begeisterung für das ungewöhnliche Projekt, die sehr klare Darstellung, die ausführliche Einbettung in Bestehendes, die spannenden Theoriebezüge und vieles mehr, blieben am Ende der Lektüre kleinere Fragezeichen übrig. Einerseits hätte ich mir mehr konkrete Einsichten in die gewonnenen Erkenntnisse gewünscht. Häufig liegt, meiner Meinung nach, die Qualität qualitativer Forschung in der Dichte der Beschreibungen, auf die hier aber bewusst verzichtet wurde. So bleibt die eigentliche Kommunikation der Strafgefangenen im Endeffekt wenig greifbar und damit weiterhin so entrückt wie die Welt der Strafgefangenen an sich. Zudem konnte ich mich des Eindrucks nicht erwehren, dass sich das Buch zu sehr auf Verteidigung fokussiert und dabei an mancher Stelle die Stärken und Potenziale der 
Studie aus dem Blick geraten und nicht voll ausgeschöpft werden. Die Ausführlichkeit der Einordnung nicht nur der sozialkonstruktivistischen Perspektive, sondern auch des Vorgehens bei der Typenbildung, des Verzichts auf Handlungsanweisungen, der Konzentration auf den kommunikativen Kosmos statt auf Mediennutzung (was wiederum der sozialkonstruktivistischen Perspektive geschuldet ist) und anderes mehr - all das wird so gründlich ausgeführt, dass die eigentlichen Erkenntnisse etwas ins Hintertreffen geraten. Der Schutzwall des Strafvollzugs wurde, so scheint es, so sehr respektiert, dass die Einblicke zum Teil hinter just diesem Wall verbleiben. Den Wert der Erkenntnisse als solcher mindert dies aber nicht - die Lektüre des Buches lohnt sich sehr.

Funding Open Access funding provided by Projekt DEAL.

Open Access Dieser Artikel wird unter der Creative Commons Namensnennung 4.0 International Lizenz veröffentlicht, welche die Nutzung, Vervielfältigung, Bearbeitung, Verbreitung und Wiedergabe in jeglichem Medium und Format erlaubt, sofern Sie den/die ursprünglichen Autor(en) und die Quelle ordnungsgemäß nennen, einen Link zur Creative Commons Lizenz beifügen und angeben, ob Änderungen vorgenommen wurden.

Die in diesem Artikel enthaltenen Bilder und sonstiges Drittmaterial unterliegen ebenfalls der genannten Creative Commons Lizenz, sofern sich aus der Abbildungslegende nichts anderes ergibt. Sofern das betreffende Material nicht unter der genannten Creative Commons Lizenz steht und die betreffende Handlung nicht nach gesetzlichen Vorschriften erlaubt ist, ist für die oben aufgeführten Weiterverwendungen des Materials die Einwilligung des jeweiligen Rechteinhabers einzuholen.

Weitere Details zur Lizenz entnehmen Sie bitte der Lizenzinformation auf http://creativecommons.org/ licenses/by/4.0/deed.de.

Dr. Maren Hartmann ist Professorin für Kommunikations- und Mediensoziologie an der Universität der Künste Berlin. 